\title{
Edge solitons of topological insulators and fractionalized quasiparticles in two dimensions
}

\author{
Dung-Hai Lee, ${ }^{1,2}$ Guang-Ming Zhang, ${ }^{3}$ and Tao Xiang ${ }^{4,5}$ \\ ${ }^{1}$ Department of Physics, University of California at Berkeley, Berkeley, CA 94720, USA \\ ${ }^{2}$ Material Science Division, Lawrence Berkeley National Laboratory, Berkeley, CA 94720, USA \\ ${ }^{3}$ Department of Physics, Tsinghua University, Beijing 100084, China \\ ${ }^{4}$ Institute of Physics, Chinese Academy of Sciences, P. O. Box 603, Beijing 100080, China \\ ${ }^{5}$ Institute of Theoretical Physics, Chinese Academy of Sciences, P.O. Box 2735, Beijing 100080, China
}

(Dated: August 7, 2021)

\begin{abstract}
An important characteristic of topological band insulators is the necessary presence of in-gap edge states on the sample boundary. We utilize this fact to show that when the boundary is reconnected with a twist, there is always zero-energy defect states. This provides a natural connection between novel defects in the two-dimensional $p_{x}+i p_{y}$ superconductor, the Kitaev model, the fractional quantum Hall effect, and the one-dimensional domain wall of polyacetylene.
\end{abstract}

PACS numbers: 73.43.-f, 71.10.Li

Excitations carrying fractional quantum numbers (e.g. fractional charge), such as the quasiparticles in the fractional quantum Hall effect [1], have always been an subject of interest. In 1976 Jackiw and Rebbi [2] wrote a seminal paper which laid the foundation of charge fractionalization in one spatial dimension. Four years later the influential paper of Su, Schrieffer and Heeger [3] proposes the "Jackiw-Rebbi soliton" as the charge carrier in doped polyacetylene. Today, all quantum number fractionalization phenomena, such as the fractionalization of magnon into spinons [4], can be attributed to the JackiwRebbi mechanism.

In the last fifteen years, starting with the fractional quantum Hall effect, condensed matter physicists stumbled upon several instances where quantum number fractionalization occurs in two spatial dimensions. These include the quasiparticles of the "Pfaffian" quantum Hall state [5], the vortices of a spin-polarized $p_{x}+i p_{y}$ superconductor [6, 7, 8, 9, 10], and the topological excitations in a spin model proposed by Kitaev [11]. However, what is lacking is a general framework, like the JackiwRebbi theory in one dimension, specifying the condition under which fractionalized excitations will appear. In this paper we provide such a mechanism and reveal its connection to the Jackiw-Rebbi theory. In particular, we show that, in two dimensions, fractional charge will naturally appear around defects of "topological" insulators [12, 13, 14].

Recently it has been shown that the existence of fractional charge in the quantum Hall effect is connected to the existence of fractionally charged domain wall in certain one dimensional systems [15, 16, 17, 18]. In the following we generalize such connection and show that when the boundary of a topological insulator, an insulator which necessarily possesses in-gap edge state, are reconnected with a twist, there is always zero-energy defect states possessing fractional quantum number.

Let us begin by considering the Kitaev model [11].
This exactly soluble model describes a honeycomb lattice of quantum one-half spins interacting via three type of nearest-neighbor interactions (Fig. 1a). The Hamiltonian is given by

$$
H=\frac{1}{2} \sum_{n \in \mathrm{w}} \sum_{\mu=1,2,3} J_{\mu} \sigma_{n+e_{\mu}}^{\mu} \sigma_{n}^{\mu},
$$

where "w/b" abbreviates for the white/black sublattice (see Fig. 1 1 ), and $n+e_{\mu}$ is the nearest neighbors of $n$ along the $\mu$ bond, $\sigma^{\mu}$ are the Pauli matrices. By performing Jordan-Wigner transformation it was shown in Ref. 19] that this model is equivalent to a free Majorana fermion model:

$$
H_{M}=-i \sum_{n \in \mathrm{w}}\left\{\sum_{\mu=1,2} J_{\mu} \gamma_{n+e_{\mu}} \gamma_{n}+J_{3} D_{n} \gamma_{n+e_{3}} \gamma_{n}\right\},
$$

where $D_{n}= \pm 1$ is a classical Ising variable and $\gamma_{n}$ 's are Majorana fermion operators [19]. Since the honeycomb lattice is consisted of two sublattices, Eq. (2) can be recast into

$$
H_{M}=\sum_{n \in \mathrm{w}, m \in \mathrm{w}} \Psi_{n}^{\dagger} H_{n m} \Psi_{m}
$$

where $\Psi_{n}^{\dagger}=\left(\gamma_{n}, \gamma_{n+e_{3}}\right)$. In Eq. (3) the $2 \times 2$ coupling matrix $H_{n m}$ has value: $H_{n m}=J_{3} D_{n} \sigma_{2}$ for $n=m$, and $H_{n m}=\mp\left(i J_{\mu} / 2\right) \sigma_{ \pm}$for $n \neq m$. The upper sign applies if the bond linking $m$ to $n$ is a black-to-white bond, and the lower sign applies if it is a white-to-black bond.

In the ground state, the classical Ising variable takes value $D_{n}=1$ modulo a global flip per row [19]. For $D_{n}=$ $1, H_{M}$ is translation invariant, and can be diagonalized by Fourier transformation. The Bloch matrix of $H_{M}$ is given by $H_{M}(\mathbf{k})=h_{1}(\mathbf{k}) \sigma_{1}+h_{2}(\mathbf{k}) \sigma_{2}$ with

$$
\begin{aligned}
& h_{1}(\mathbf{k})=-J_{2} \sin \alpha(\mathbf{k})+J_{1} \sin \beta(\mathbf{k}), \\
& h_{2}(\mathbf{k})=J_{3}+J_{2} \cos \alpha(\mathbf{k})+J_{1} \cos \beta(\mathbf{k}),
\end{aligned}
$$




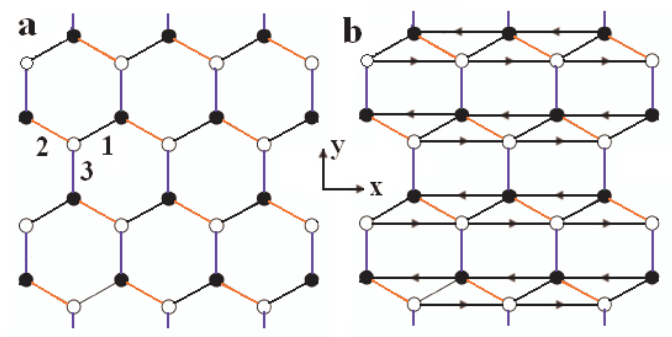

FIG. 1: (a) A graphical representation of the Kitaev model. There are two sublattices (white and black), and three types of bonds (labeled by 1,2,3, or black, red, blue). (b) The graphical representation of $H+H_{t}$. In the Majorana representation, the three-spin interactions in Eq. (5) become next neighbor hopping along the zigzag chain in the $\mathrm{x}$-direction. The arrow represents the direction in which the second neighbor hopping matrix elements are $-i J_{4}$.

and $\alpha(\mathbf{k})=\left(\sqrt{3} k_{x}-3 k_{y}\right) / 2, \beta(\mathbf{k})=\left(\sqrt{3} k_{x}+3 k_{y}\right) / 2$. It is easy to show that for $J_{1}+J_{2} \geq J_{3} \geq\left|J_{1}-J_{2}\right|$ the energy spectrum is gapless. The possible connection to the quantum spin liquids has been discussed in Ref. [20]. In Ref. [11] a magnetic field is introduced to open an excitation gap in this parameter region, and the nonAbelian quasiparticles become low energy excitations of this gapped phase.

Unfortunately the magnetic field spoils the integrability of the model. Here we propose a different way of opening a gap while maintaining the integrability. This is achieved by adding the following three-spin interaction to Eq. (11)

$$
H_{t}=\frac{J_{4}}{2} \sum_{(i j k) \in \Delta} \sigma_{i}^{2} \sigma_{j}^{3} \sigma_{k}^{1}+\frac{J_{4}}{2} \sum_{(i j k) \in \nabla} \sigma_{i}^{1} \sigma_{j}^{3} \sigma_{k}^{2} .
$$

Here $(i j k)$ denote three adjacent sites (with $i$ being the left-most one) along the zigzag chain running along the $\mathrm{x}$-direction. Depending on whether $(i j k)$ form an uppointing or a down-pointing triangle, we use the first or second term of Eq. (5). In terms of the Majorana fermion operators this amounts to adding a second nearest neighbor hopping between sites along the zigzag chain:

$$
H_{t}=-i J_{4}\left(\sum_{i, k \in \mathrm{w}} \gamma_{i} \gamma_{k}-\sum_{i, k \in \mathrm{b}} \gamma_{i} \gamma_{k}\right) .
$$

Then the Bloch matrix becomes $H_{M}(\mathbf{k})=h_{1}(\mathbf{k}) \sigma_{1}+$ $h_{2}(\mathbf{k}) \sigma_{2}+h_{3}(\mathbf{k}) \sigma_{3}$, where $h_{3}(\mathbf{k})=2 J_{4} \sin \left(\sqrt{3} k_{x}\right)$. The vector function $\mathbf{h}(\mathbf{k})$ is a continuous mapping from the first Brillouin zone to the space spanned by $\mathbf{h}=$ $\left(h_{1}, h_{2}, h_{3}\right)$. The image is a closed two-dimensional manifold (henceforth referred as the $h$-surface). Since the eigenvalues of the Block matrix are $\pm|\mathbf{h}(\mathbf{k})|$, it follows that if the $h$-surface contains the origin, the spectrum is gapless, otherwise the spectrum has a gap.

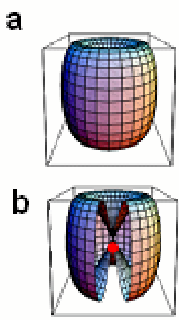

c
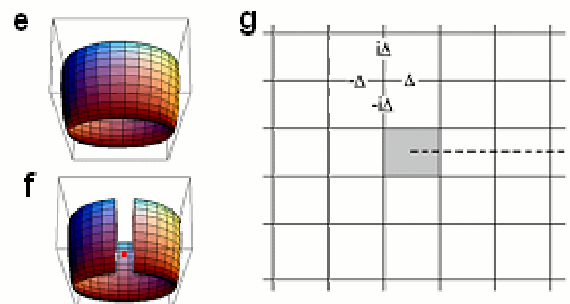

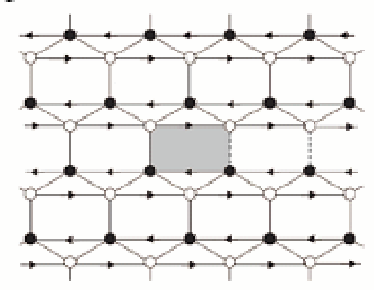

d

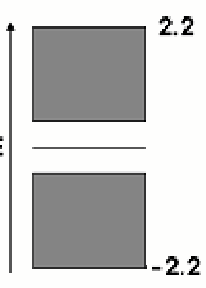

h

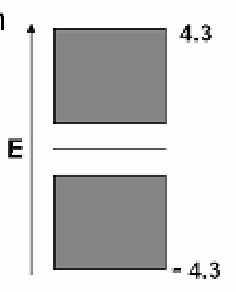

FIG. 2: The $h$-surface for the Kitaev model (a) and the $p_{x}+i p_{y}$ superconductor (e). In panels (b) and (f) the $h$ surfaces are dissected to expose the origin (red dot). (c) Topological defects in the Kitaev model. To the right of the greyshaded plaquette the sign of $D_{n}$ 's are reversed. As a result the corresponding vertical hopping matrix elements change sign. They are shown by the dashed bonds. (d) The eigenspectrum assoicated with two far separated topological defects in the Kitaev model with $J_{1}=J_{2}=1, J_{3}=0.2$ and $J_{4}=0.5$. (g) A vortex centered at the gray-shaded plaquette in a $p_{x}+i p_{y}$ superconductor. The pairing order parameter is shown for four bonds. (h) The energy spectrum of two far-separated vortex-antivortex pair with $t=1, \Delta_{0}=0.5$ and $\mu=0.3$.

For a $h$-surface not containing the origin, there is an integer topological index

$$
\mathcal{P}=\frac{1}{8 \pi} \int d^{2} k \epsilon^{\mu \nu} \hat{\mathbf{h}} \cdot\left(\partial_{k_{\mu}} \hat{\mathbf{h}} \times \partial_{k_{\nu}} \hat{\mathbf{h}}\right)
$$

which counts the number of times the unit vector $\hat{\mathbf{h}}$ wraps around the origin. As shown in Ref. 21, $\mathcal{P}$ is proportional to the well known "TKNN" index 22] in the case of two bands. Spectra characterized by different $\mathcal{P}$ are topologically distinct. They can not be deformed into each other without gap closing. In the parameter regime where non-abelian quasiparticle exists $\mathcal{P}=1$.

Topological excitations of the Kitaev model are created by reversing the sign of $D_{n}$ 's in $H_{M}$ along half a row. This is shown by the dashed bonds in Fig. (2r). In Fig. (2 d) we have shown the result of numerical diagonalization for a system of 1600 sites with toric boundary condition. Because of the boundary condition two defects are introduced, they are separated by 100 sites in the $\mathrm{x}$-direction. They introduce two mid-gap states with a tunnel-splitting (which is already invisible here) which decreases exponentially with the separation.

Now we switch gear to discuss the vortices in a spin-polarized $p_{x}+i p_{y}$ superconductor. Let us consider this problem on a square lattice. Similar to the Kitaev 
model, the Hamiltonian can also be written in the form of Eq. (3), except $\Psi_{n}^{\dagger}=\left(c_{n}^{\dagger}, c_{n}\right)$ and $c_{n}$ is a fermion operator. The Bloch matrix is characterized by $\mathbf{h}(\mathbf{k})=$ $\left(-\Delta_{0} \sin k_{y}, \Delta_{0} \sin k_{x},-t\left(\cos k_{x}+\cos k_{y}\right)-\mu\right)$. Here $\Delta_{0}$ is the pairing amplitude, $t$ is the hopping integral, and $\mu$ is the chemical potential. The $h$-surface is shown in Fig. (2f) and (2f). Straightforward calculation shows that $\mathcal{P}=1$. After a singular gauge transformation, a vortex can be created by reversing the sign of the hopping matrix elements along a cut as shown in Fig. (2r). Explicit calculation shows that there is also a zero mode associated with each vortex (Fig. 2h h).

For each free Majorana or Bogoliubov fermion model discussed above, there is a free fermion model with identical excitation spectrum. To obtain this fermion model we simply replace $\Psi_{n}^{\dagger}$ in Eq. (3) by the fermion operator $\Psi_{n}^{\dagger}=\left(c_{1 n}^{\dagger}, c_{2 n}^{\dagger}\right)$ where 1 and 2 are "flavor" indices (they might represent the two sites in the unit cell of a lattice). This fermion model acts as a representative of all models which share the same $H_{n m}$ (hence the same eigen spectrum). However, while the representative fermion model is global U(1) invariant, the Majorana and Bogoliubov fermion models only have $Z_{2}$ symmetry. The fact that in the $Z_{2}$ models the particle number is only conserved modulo two is the root of non-abelian statistics. In the rest of the discussion, we refer to the system described by a gapped free fermion model with non-zero $\mathcal{P}$ as a "topological band insulator". Thus the representative fermion models for the Kitaev model and the $p_{x}+i p_{y}$ superconductor are topological band insulators. Knowing the properties of edge states and defects of the representative fermion model, one can readily deduce the corresponding properties of the Majorana fermion (Kitaev) or the Bogoliubov fermion $\left(p_{x}+i p_{y}\right)$ models with the same $H_{n m}$. For example, while in the fermion model the edge states are free fermions, and the defect zero modes carry half fermion quantum numbers, those in the Majorana/Bogoliubov fermion model are true Majorana fermions.

In the following we provide an unifying mechanism for the appearance of defect zero mode when the representative fermion model describes a topological band insulator. As an example, let us consider the fermion representative of the Kitaev model (Fig. 3a a). Fig. (3b) shows the gapped spectrum as a function of momentum along the longitudinal circle. Due to its topological nature, if we remove a row of bonds (Fig. 3r), in-gap edge states appear 23. as shown in Fig. (3d). The left and right moving chiral edge fermions, represented by the arrowed red lines in Fig. (3r), are described by a massless Dirac Hamiltonian in 1D. If we reconnect the two edges, but with weaker bonds, a smaller gap reappear in the edge spectrum (see Fig. [3e and Fig. 3f). The edge fermions are now described a .
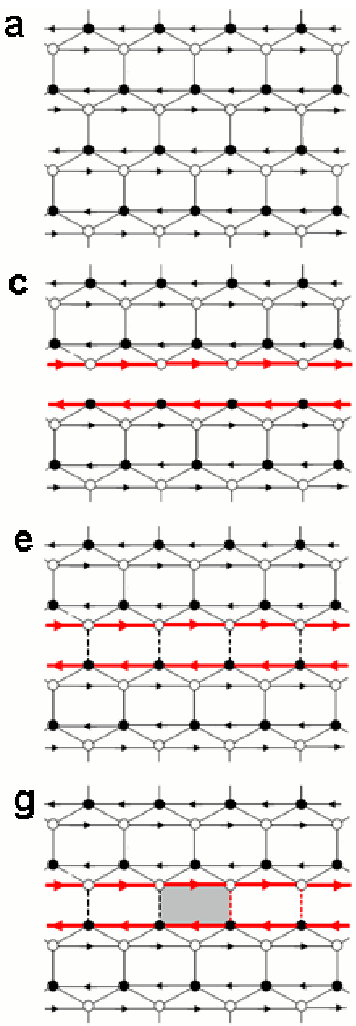

b

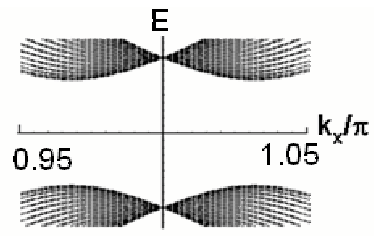

d

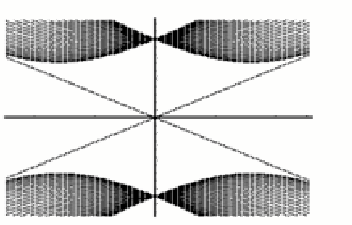

f

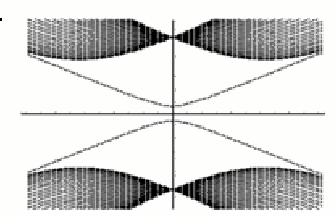

h

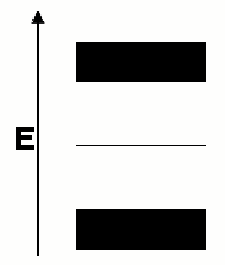

FIG. 3: (a) The Kitaev model and (b) its eigen-spectrum as a function of wavevector $k_{x}$ along the x-direction around $k_{x}=\pi$. (c) One row of bonds along $\hat{x}$ are removed and (d) the corresponding energy spectrum. The arrowed-red lines in (c) indicate the edge states are chiral. (e) A weaker hopping between the edge (dashed black vertical bonds) is reintroduced. (f) The gapped energy spectrum corresponds to (e). (g) An edge soliton (marked by the gray plaquette) is introduced by reversing the sign for half of the vertical bonds (red dashed lines) between the edges. (h) The energy spectrum corresponds to (f). The spectra in (b), (d) and (h) are obtained from $H_{F}$ with $J_{1}=J_{2}=1, J_{3}=0.2$ and $J_{4}=0.5$. The spectrum in (f) and (h) was obtained with a restored edge coupling $J_{3}= \pm 0.02$.

by a massive Dirac Hamiltonian

$$
H_{E}=\int d x\left(-i v \psi^{\dagger} \sigma_{z} \partial_{x} \psi+m \psi^{\dagger} \sigma_{x} \psi\right)
$$

where $v$ is the edge velocity, $\psi^{\dagger}=\left(\psi_{R}^{\dagger}, \psi_{L}^{\dagger}\right)$ with $\psi_{R / L}^{\dagger}$ being the right/left fermion creation operators. When the restored bonds have a sign reversal (Fig. 3 $\mathrm{k}$ ), the mass term in Eq. (8) becomes X-dependent and changes sign as $x$ goes through the location of the topological defect. This should result in one localized zero mode per defect according to Ref.[2, 3]. Fig. (3h) shows that this is indeed true. The presence of such zero mode plus the fact that the spectra of Majorana fermion models are $E \leftrightarrow-E$ symmetric, immediately imply that the zero modes are Majorana fermion states in the original Kitaev model. Furthermore, using the argument of Ref. 24] it 


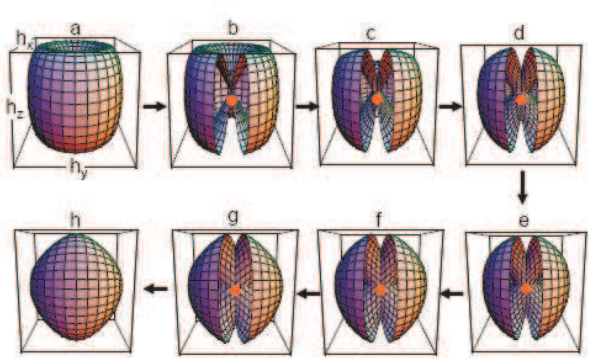

FIG. 4: The evolution of the of the $h$-surface as the model in Fig. (1) is gradually deformed into Haldane's model. In constructing the figure we used $J_{1}=J_{2}=J_{3}=1$. The full strength of the second neighbor hopping is 0.5 .

can be shown that the braiding of such Majorana fermion defects leads to the non-Abelian statistics. Thus, via the mechanism of Refs. 2, 3] a two dimensional defect with fractionalized quantum number has emerged! Its presence is determined by the topological nature of the host bulk band insulator just as the edge states are.

Actually, a similar phenomenon was also found in the continuum theory of Dirac fermions interacting with the topological defects of a Higgs field 25]. However, in all the examples we considered here, the location of the edge Dirac point in the momentum space is far away from those of the bulk Dirac points. Consequently the theory discussed in Ref. [25] is not applicable here.

As a digression, we now show the fermion representative of the Kitaev model is topologically equivalent to Haldane's lattice model for integer quantum Hall effect [12]. Fig. (44) shows the evolution of the $h$-surface by gradually switching off the second neighbor hopping in Haldane's model which are not contained in Eq. (6). The leftmost column are the $h$-surfaces for the Haldane model (top) and Kitaev (bottom), respectively. In the rest of the figure the surfaces are dissected to reveal the origin (the red dot). Clearly as we follow the evolution the origin never migrate across the $h$-surface. Thus $\mathcal{P}$ for the two models are the same.

Finally what about the Laughlin quasiparticles? Although fractional quantum Hall liquids are not band insulators they are clearly topological insulators. Indeed, as shown by Wen [26], when a quantum Hall liquid on a torus is cut open (Fig. 3a), there are "chiral Luttinger liquid" in-gap edge modes. At $1 / m$ filling, the edge modes are described by the following free boson Hamiltonian

$$
H_{B}=\int d x\left\{\frac{2 \pi}{m} \Pi(x)^{2}+\frac{m}{8 \pi}\left[\partial_{x} \phi(x)\right]^{2}\right\},
$$

where $\Pi$ and $\phi$ are conjugate boson fields satisfying $[\Pi(x), \phi(y)]=i \delta(x-y)$. To reconnect the edges, a poten- tial $V=-g \int d x \cos (m \phi)$ needs to be added [26]. When $g$ is sufficiently big, a gap opens in the edge spectrum and the ground states become $m$-fold degenerate. They are characterized by $\langle\phi(x)\rangle=2 \pi l / m(l=0, \ldots, m-1)$. In this case an edge soliton is where $\phi(x)$ interpolates between two different ground states, say, $\langle\phi(x)\rangle=0$ and $2 \pi / m$. As shown by Goldstone and Wilczek [27], such a soliton carries a charge $\Delta Q=\Delta \phi / 2 \pi=1 / m$, precisely the same as a that of a Laughlin quasiparticle [1]. Hence the Laughlin quasiparticles are also edge solitons of a topological insulator!

Acknowledgement DHL was supported by DOE Contract No. DE-AC02-05CH11231. GMZ and TX acknowledge the support from the NSF-China and the national program for basic research.

[1] R. B. Laughlin, Phys. Rev. Lett. 50, 1395 (1983).

[2] R. Jackiw and C. Rebbi, Phys. Rev. D 13, 3398 (1976).

[3] W. P. Su, J. R. Schrieffer and A. J. Heeger, Phys. Rev. B 22, 2099 (1980).

[4] F. D. M. Haldane, Phys. Rev. Lett. 66,1529 (1991).

[5] G. Moore and N. Read, Nucl. Phys. B 360, 362 (1991).

[6] N. B. Kopnin and M. M. Salomaa, Phys. Rev. B 44, 9667 (1991).

[7] G. E. Volovik, JETP Lett. 70, 609 (1999).

[8] N. Read and D. Green, Phys. Rev. B 61, 10267 (2000).

[9] M. Stone and R. Roy, Phys. Rev. B 69, 184511 (2004).

[10] S. Tewari, S. Das Sarma and D.-H. Lee, Phys. Rev. Lett. 99, 037001 (2007).

[11] A. Kitaev, Ann. Phys. 321, 2 (2006).

[12] F. D. M. Haldane, Phys. Rev. Lett. 61, 2015 (1988).

[13] C. L. Kane and E. J. Mele, Phys. Rev. Lett. 95, 226801 (2005).

[14] S. Murakami, N. Nagaosa and S.C. Zhang, Science 301, 1348 (2003).

[15] A. Seidel et al, Phys. Rev. Lett. 95, 266405 (2005).

[16] A. Seidel and D.-H. Lee, Phys. Rev. Lett. 97, 056804 (2006).

[17] E. J. Bergholtz and A. Karlhede, Phys. Rev. Lett. 94, 026802 (2005).

[18] E. J. Bergholtz et al, Phys. Rev. B 74, 81308 (2006).

[19] X.-Y. Feng, G.-M. Zhang and T. Xiang, Phys. Rev. Lett. 98, 087204 (2007).

[20] G. Baskaran, S. Mandal, and R. Shankar, Phys. Rev. Lett. 98, 247201 (2007).

[21] W.-Y. Hsiang and D.-H. Lee, Phys. Rev. A 64, 052101 (2001).

[22] D. J. Thouless et al, Phys. Rev. Lett. 49, 405-408 (1982).

[23] Y. Hatsugai, Phys. Rev. Lett. 71, 3697-3700 (1993).

[24] D. A. Ivanov, Phys. Rev. Lett. 86, 268 (2001).

[25] C. G. Callan Jr. and J. A. Harvey, Nucl. Phys. B 250, 427 (1985).

[26] X. G. Wen, Phys. Rev. B41, 12838-12844 (1990).

[27] J. Goldstone and F. Wilczek, Phys. Rev. Lett. 47, 986989 (1988). 